\title{
Antimicrobial resistance patterns in outpatient urinary tract infections - the constant need to revise prescribing habits
}

\author{
Fredricka J Bosch, Cloete van Vuuren, Gina Joubert
}

Background. There is a global emergence of resistance against commonly prescribed antibiotics. Empirical antibiotic prescribing should be guided by local antimicrobial susceptibility patterns.

Aim. To identify organisms and determine antibiotic susceptibility in urinary tract infections (UTIs) at 3 Military Hospital, Bloemfontein.

Methods. All urine samples collected during 2008 were analysed. The first positive urine culture per patient collected from the casualty, gynaecology, internal medicine and surgical outpatient departments were included. Only adult patients ( $>12$ years old) were included. Prior use of antibiotics and underlying conditions were determined from electronic and paper-based patient and pharmacy records.

Results. Positive cultures $(N=65)$ were divided into uncomplicated $(N=28)$ and complicated $(N=37)$ UTIs. Escherichia coli $($ E. coli) was the most common uropathogen in uncomplicated $(75 \%)$ and complicated (59\%) UTIs. In uncomplicated UTIs, trimethoprim- sulfamethoxazole (TMP-SMX) (54\%) and amoxicillin (46\%) had the highest rates of resistance. Nitrofurantoin and ciprofloxacin had sensitivity rates of $89 \%$. Co-amoxiclav was most commonly prescribed (36\%). In complicated UTIs, TMP-SMX (68\%) and amoxicillin $(65 \%)$ had the highest resistance rates, followed by ciprofloxacin (41\%). Nitrofurantoin had a sensitivity rate of $73 \%$. Ciprofloxacin was prescribed most often (35\%). All E. coli UTI isolates were sensitive to nitrofurantoin.

Conclusion. E. coli remains the most common uropathogen. TMPSMX and amoxicillin are of no value in this population with UTIs. Uncomplicated UTIs can be treated effectively with nitrofurantoin; this will lead to cost savings and sparing quinolones as a class of antibiotics known to induce resistance. In this setting, ciprofloxacin should not be used empirically for complicated UTIs.

S Afr Med J 2011;101:328-331.
Uncomplicated urinary tract infections (UTIs) are usually diagnosed in previously healthy women with no underlying disease or anatomical anomalies, whereas complicated UTIs refer to patients with co-morbidities, anatomical anomalies, and catheter-associated or nosocomially acquired infections. ${ }^{1}$ UTIs are a global financial burden, and the emergence of resistance in uncomplicated and complicated uropathogens is of great concern. ${ }^{2}$

Escherichia coli (E. coli) is the most common uropathogen in both uncomplicated and complicated UTIs. ${ }^{1}$ There is a wide spectrum of pathogens causing complicated UTIs including E. coli, Klebsiella, Pseudomonas, Enterobacter, Enterococcus and Candida species. ${ }^{1,3}$

Guidelines recommend a short course of trimethoprimsulfamethoxazole (TMP-SMX) for the empiric treatment of uncomplicated UTIs if resistance is less than $20 \%{ }^{5-7}$ In South Africa, TMP-SMX is widely used for UTI prophylaxis and treatment, and for Pneumocystis jirovecii prophylaxis in immunocompromised patients, which has led to increased bacterial resistance. ${ }^{8}$ In a setting where more than $20 \%$ of $E$. coli cultures are resistant to TMP-SMX, guidelines recommend ciprofloxacin, nitrofurantoin or fosfomycin. ${ }^{6}$ Kallen $e t$ al. were concerned about the increased use of quinolones causing increased resistance to this class of antibiotics. ${ }^{9}$ Worldwide changes in susceptibility of $E$. coli have occured, and the prevalence of community-acquired extended-spectrum beta-lactamase (ESBL) producing organisms has increased..$^{10-12}$

Department of Internal Medicine, University of the Free State, Bloemfontein Fredricka J Bosch, MB ChB, Dip HIV Man (SA)

Cloete van Vuuren, MB ChB, MFam Med, MMed (Int)

Department of Biostatistics, University of the Free State

Gina Joubert, BA, MSc
Surveillance data must be regularly collected for a specific infection to guide antibiotic use. ${ }^{3}$ Prior antibiotic exposure must be kept in mind when deciding on empiric antibiotics as this is a strong risk factor for resistant E. coli UTIs. ${ }^{13}$

\section{Methods}

A retrospective survey of antimicrobial susceptibility data and antibiotic prescribing behaviour for adult ( $>12$ years old) outpatient UTIs at 3 Military Hospital, Bloemfontein, was done.

Resistance patterns may differ depending on the geographic area, and have not previously been documented for UTIs in this institution. Our objectives were to identify the aetiological agents associated with uncomplicated and complicated UTIs, and the rate of resistance, and design appropriate empiric evidence-based antibiotic guidelines for this institution.

Permission for the study was granted by the Hospital Administrator and the Ethics Committee of the Faculty of Health Sciences, University of the Free State.

Members and retired members and their families of the South African National Defence Force (SANDF) are seen exclusively by military-appointed doctors, and medication dispensed by military pharmacies. All encounters are captured in an electronic database. A list of all urine samples sent from 3 Military Hospital from January to December 2008 was compiled from the DISA system by the National Health Laboratory Service (NHLS).

Positive urine cultures collected from the casualty, gynaecology, internal medicine and surgical outpatient departments were included. If more than one urine microscopy, culture and sensitivity (MC\&S) specimen was sent during a specific UTI episode, only the first specimen was included.

Electronic database and paper-based patient records were used to compile a list of co-morbidities, antibiotic use during the previous year, and antibiotics prescribed for the UTI episode under investigation.

Uncomplicated UTIs were defined as the first episode of UTI in outpatients with no underlying anatomical anomalies and no indwelling devices. For this study, the first UTI in pregnant women 
and in diabetic patients was regarded as uncomplicated. Patients with complicated UTIs were defined as: males (including BPH, prostatitis, epididymo-orchitis, prostate cancer), following a recent cystoscopy, with indwelling urinary catheters/intermittent self-catheterisation, recurrent UTIs in females (defined as $\geq 3$ culture-positive UTIs per year), urological abnormalities, pyelonephritis, renal failure, renal stones, UTIs with multiple organisms, and UTIs in patients who were recently hospitalised.

Cultures positive for Candida species and cultures with mixed growth were excluded. Mixed growth was defined as a growth of more than one organism in a midstream urine sample indicative of contamination. If it was a sterile urine sample (e.g. supra-pubic catheter sample), multiple organisms were reported as pathogenic.

\section{Results}

Fig. 1 illustrates the selection process used to isolate the first positive culture per patient $(N=65)$ from the 791 samples sent for MCS during 2008. These samples were divided into uncomplicated $(N=28)$ and complicated $(N=37)$ UTIs.

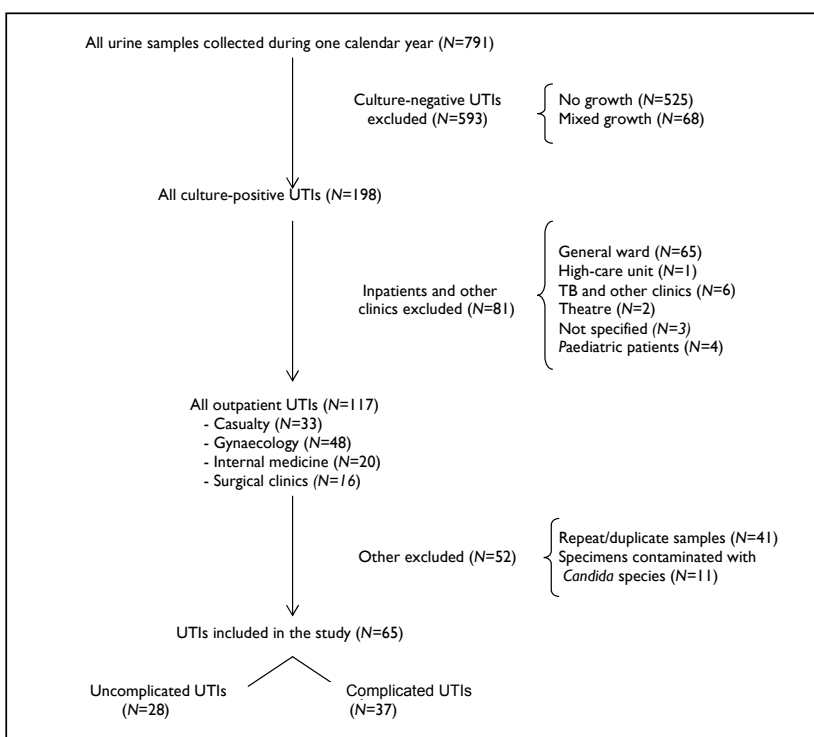

Fig. 1. Selection process used to determine isolated outpatient urinary tract infections (UTIs).

In the complicated group, 54\% $(N=20)$ were female and $46 \%$ $(N=17)$ male. The age distribution for both groups is illustrated in Fig. 2. No growth was recorded in $66 \%$ of samples $(N=525)$. Sixtyeight samples had mixed growth.

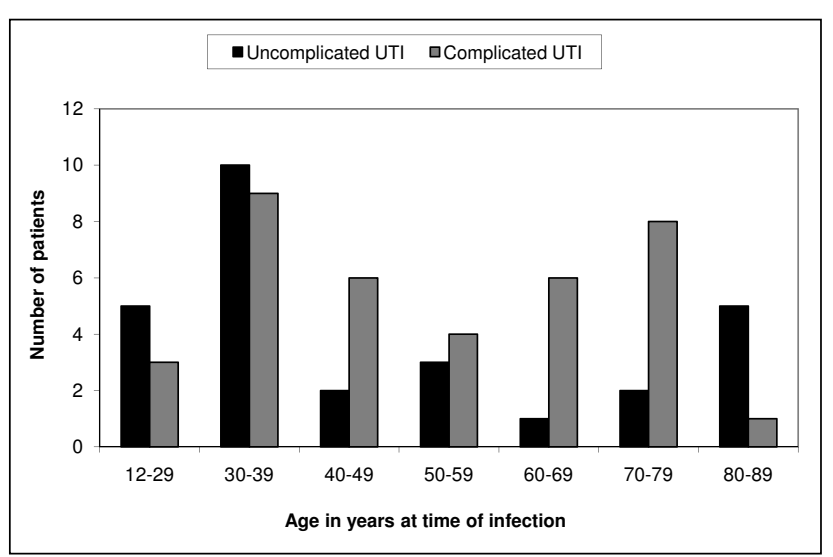

Fig. 2. Age distribution of patients.

\section{Uncomplicated UTIs}

E. coli was responsible for $75 \%(N=21)$, Enterococcus faecalis (E. faecalis), Proteus mirabilis and Streptococcus agalactiae for $7 \%(N=2)$ each, and Klebsiella pneumoniae for one episode of uncomplicated UTI in this population. There were no cases of extended spectrum $\beta$-lactamase (ESBL) producing organisms.

\section{For $E$. coli isolates only}

Further analysis of the $E$. coli isolates $(N=21)$ showed sensitivity to nitrofurantoin and cefuroxime in all 21 cultured cases. Amoxicillin and TMP-SMX had the highest resistance rate $(57 \%, N=12$ and $52 \%$, $N=11$ respectively), followed by ciprofloxacin and co-amoxiclav with $5 \%(N=1)$ each. One sample had only intermediate sensitivity to co-amoxiclav (Fig. 3). Co-amoxiclav was the most frequently prescribed antibiotic $(38 \%, N=8)$, followed by ciprofloxacin $(19 \%, N=4)$, amoxicillin $(14 \%, N=3)$, cefuroxime $(10 \%, N=2)$ and nitrofurantoin $(5 \%, N=1)$. TMP-SMX was never prescribed, and 3 patients did not have any record of antibiotics dispensed.

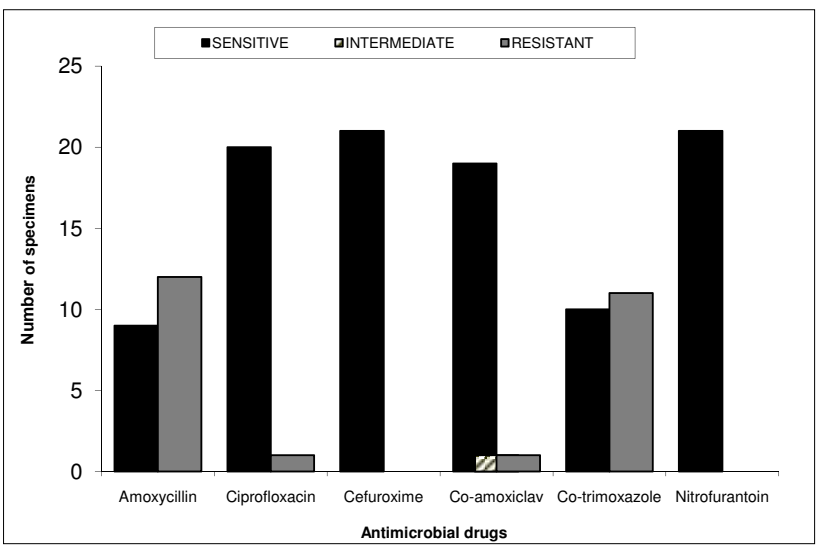

Fig. 3. Resistance to antibiotics in uncomplicated UTIs for E. coli isolates only $(\mathrm{N}=21)$.

\section{For all uncomplicated UTIs}

Analysis of all uncomplicated UTIs $(N=28)$ showed resistance to co-amoxiclav in only $4 \%(N=1)$. TMP-SMX and amoxicillin had the highest resistance rates of $54 \%(N=15)$ and $46 \%(N=13)$ respectively, followed by cefuroxime $14 \%(N=4)$ and ciprofloxacin and nitrofurantoin with $11 \%(N=3)$ resistance (Fig. 4). Co-amoxiclav was the most frequently prescribed antibiotic $(36 \%, N=10)$, followed by ciprofloxacin $(18 \%, N=5)$, amoxicillin $(11 \%, N=3)$, cefuroxime and TMP-SMX ( $7 \%$ each, $N=2)$ and nitrofurantoin $(4 \%, N=1)$. One patient received azithromycin, and 4 patients did not receive any

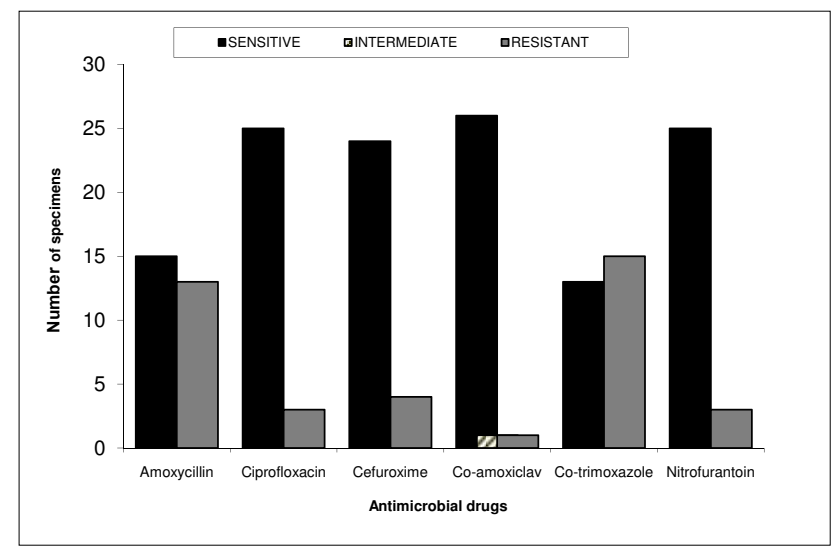

Fig. 4. Resistance to antibiotics in all uncomplicated UTIs $(\mathrm{N}=28)$. 
antibiotics. Treatment durations were in most cases significantly longer than the recommended 3-day course: 5 days (46\%), 7 days (7\%), and 10 days (7\%).

The prescribed antibiotic was appropriate in $75 \%$ of all uncomplicated UTIs according to the antibiogram.

Of females with uncomplicated UTIs, $25 \%$ were older than 65 years and $39 \%(N=11)$ were pregnant. Samples were mostly sent during the first trimester $(N=8)$. Concomitant problems included: diabetes mellitus ( $N=3)$, HIV-positive $(N=4)$, breast cancer $(N=1)$, cervical intraepithelial neoplasia $(\mathrm{CIN})$ lesion $(N=1)$, Alzheimer's disease $(N=1)$, recent episode of vaginitis $(N=2)$, intra-uterine device $(N=2)$ and incompetent cervix $(N=1)$.

Fifty-four per cent $(N=15)$ of patients had had recent antibiotic exposure unrelated to UTIs.

Complications following uncomplicated UTIs were late re-infections $(N=3)$, one Citrobacter freundii and 2 E. coli urinary tract infections. One of these patients had 2 positive urinary cultures with an ESBL-producing E. coli and E. faecalis infection within the following 2 months. Two patients cultured a different organism in the next 7 - 10 days after their first positive urine culture.

\section{Complicated UTIs}

For complicated UTIs, E. coli was responsible for 59\% $(N=22)$, Enterococcus cloacae, E. faecalis and K. pneumoniae 8\% each $(N=3)$ and Streptococcus agalactiae for $5 \%(N=2)$ of UTIs. Each of the following was responsible for one episode: Acinetobacter baumanii, Citrobacter freundii, coagulase negative staphylococcus, Enterococcus spp, Morganella morganii, Pseudomonas aeruginosa, Proteus mirabilis, Streptococcus spp, Staphylococcus epidermidis, Staphylococcus haemolyticus and Staphylococcus saprophyticus.

Two E. cloacae and one E. coli isolates were ESBL producers.

A single organism was isolated in $84 \%$ of complicated UTIs $(N=31)$. Five of the remaining urine samples cultured 2 organisms, and 1 urine sample cultured 3 organisms.

\section{For E. coli isolates only}

Analysis of the antibiograms of the E. coli isolates $(N=22)$ showed that nitrofurantoin had a $100 \%$ sensitivity rate. TMP-SMX and amoxicillin had the highest resistance rates $(82 \%, N=18$ and $73 \%, N=16$ respectively), followed by ciprofloxacin $(45 \%, N=10)$, and cefuroxime and co-amoxiclav with $9 \%(N=2)$ each (Fig. 5). Ciprofloxacin was the most frequently prescribed antibiotic $(29 \%, N=6)$, followed by nitrofurantoin and co-amoxiclav (14\%, $N=3)$, amoxicillin and TMPSMX both at $10 \%(N=2)$, and cefuroxime at $5 \%(N=1)$. Twenty-nine per cent $(N=6)$ of the patients did not receive any antibiotics.

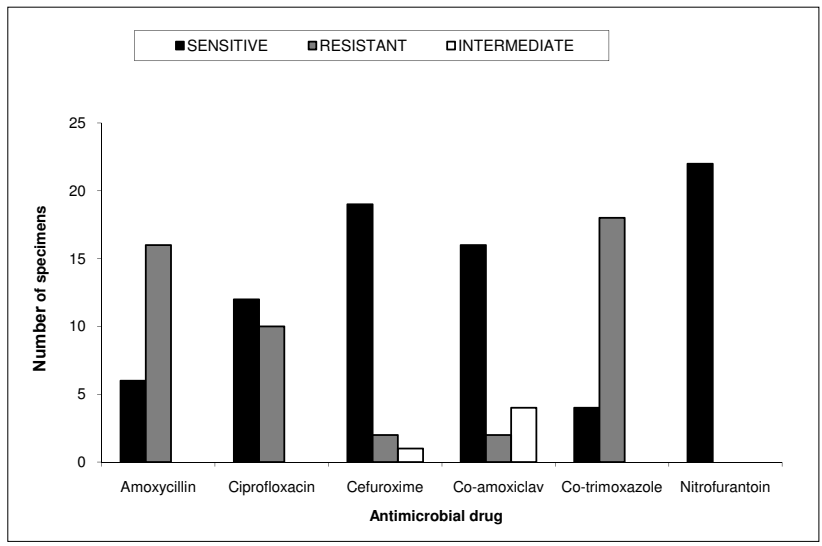

Fig. 5. Resistance to antibiotics in complicated UTIs for E. coli isolates only $(\mathrm{N}=22)$.

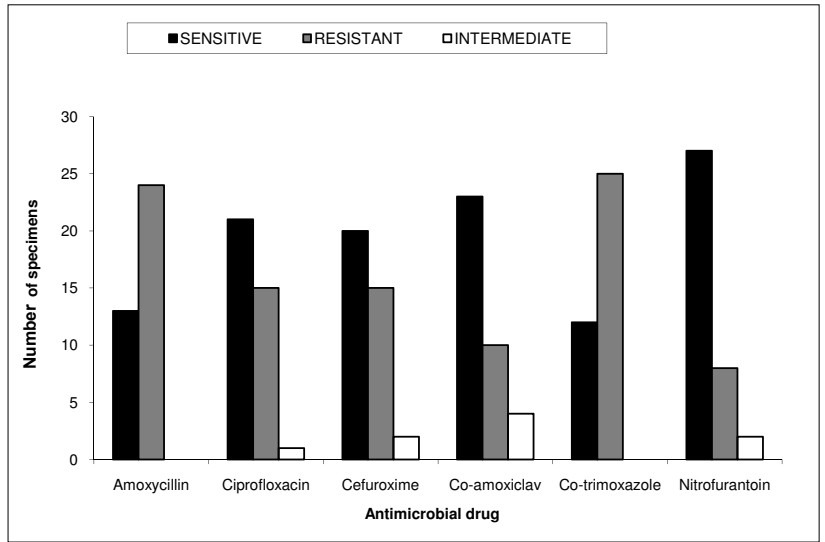

Fig. 6. Resistance to antibiotics in all complicated UTIs $(\mathrm{N}=37)$.

\section{For all complicated UTIs}

Of all complicated UTIs $(N=37)$, nitrofurantoin was the agent with the lowest resistance rate of $22 \%(N=8)$. Two cultured organisms were only intermediately sensitive to nitrofurantoin. TMP-SMX and amoxicillin had the highest resistance rate $(68 \%, N=25$ and $65 \%, N=24$, respectively), followed by cefuroxime and ciprofloxacin at $41 \%(N=15)$ each and co-amoxiclav at $27 \%(N=10)$ (Fig. 6). Ciprofloxacin was the most frequently prescribed antibiotic (35\%, $N=13)$, followed by co-amoxiclav ( $19 \%, N=7)$, TMP-SMX, amoxicillin and nitrofurantoin $(11 \%, N=4)$ each, and cefuroxime at $8 \%(N=3)$. No antibiotics were prescribed in $24 \%$ of cases. Treatment durations were as follows: 1 - 3 days (5\%), 5 days (24\%), 7 days (5\%), 10 days (19\%) and not specified (22\%).

The empiric choice of antibiotics was appropriate in $46 \%(N=17)$ of all complicated UTIs.

Concomitant problems included: diabetes mellitus $(N=9)$, malignancies $(N=5)$, recurrent UTI during pregnancy $(N=3)$, HIV-positive $(N=3)$, epididymo-orchitis $(N=2)$, benign prostate hypertrophy $(N=2)$, prostatitis $(N=2)$, prostate cancer $(N=2)$, procedure-related $(N=2)$, urinary catheter-associated $(N=9)$, recurrent infections in females $(N=4)$, renal failure $(N=4)$, renal stones $(N=1)$, urological abnormalities $(N=6)$ and pyelonephritis $(N=2)$. One patient had had a previous UTI with a resistant organism.

Sixty-two per cent $(N=23)$ of the patients had antibiotic exposure in the previous year, and $19 \%(N=7)$ had been admitted during the previous year; 13 had recurrent/late re-infections, including one ESBL-producing E. coli and one ESBL-producing E. cloacae.

\section{Discussion}

The causative organisms and resistance patterns in a given population for a specific infection must be audited to guide the empirical use of antibiotics. Antibiotic choices are determined by in vitro susceptibility, adverse effects, cost, efficacy and selection pressure to limit resistant strains. With more than $20 \%$ of cultured organisms resistant to TMPSMX and amoxicillin in uncomplicated and complicated UTIs, these two antibiotics cannot be used empirically in this setting.

Nitrofurantoin is an effective urinary tract antiseptic that is not used for other kinds of infection. It does not affect antibiotic use in any other infection, and it has a resistance rate of less than $20 \%$ in this study. Therefore it should be used as first-choice treatment in uncomplicated UTIs in this hospital.

Ciprofloxacin was the most used antibiotic in complicated UTIs and the second-most used antibiotic in uncomplicated UTIs. Ciprofloxacin had resistance rates of $11 \%$ in uncomplicated and $41 \%$ in complicated UTIs. Quinolones as a class induce resistance against a wide spectrum of unrelated antibiotics. Overuse of quinolones also 
increases the incidence of methicillin-resistant S. aureus (MRSA) in hospital and community settings. ${ }^{14}$ It is also the most potent antibiotic in the second-line treatment of tuberculosis. With the high rates of tuberculosis in South Africa, inappropriate use will have farreaching consequences. We strongly advise against the empiric use of quinolones.

Our study revealed high rates of prior antibiotic use for UTIs and other unrelated infections such as upper respiratory tract infections. These prior antibiotics might have played a role in the current resistance profiles. Since this is a retrospective study, patients could have had more previous antibiotic exposure than documented. A meta-analysis showed that antibiotic use in primary care for respiratory or urinary infections leads to selection of resistance to that antibiotic, and that this would lead to increased population carriage of resistant organisms and increased use of second-line antibiotics. ${ }^{15}$ It was also reported that infection with organisms with resistance after prior antibiotic use was greatest in the month immediately after treatment, but could persist for up to 12 months. ${ }^{15}$

Evidence-based choices of antibiotics should be practised. The result will be less resistance, fewer re-consultations, cost savings and less patient morbidity and mortality. ${ }^{16}$

A very high rate of culture-negative urine samples was received at the laboratory. This might imply that samples are sent for culture inappropriately. Routine urine culture is not necessary. ${ }^{4}$ Cost savings can be effected by sending samples from patients only with both symptoms of UTI and an abnormality on urine dipstick. The dipstick strategy is most likely to be cost-effective. ${ }^{17}$ These criteria will also help to limit the unnecessary use of empiric antibiotics.

TMP-SMX and amoxicillin are of no use in this population with UTIs. Nitrofurantoin should be the empiric choice for uncomplicated UTIs.

More than $20 \%$ of cultured bacteria in complicated UTIs were resistant to ciprofloxacin, which is commonly empirically prescribed. In this setting, ciprofloxacin should not be used empirically for complicated UTIs. For complicated UTIs, prior antibiotic use and expert clinical opinion should guide the empiric use of antibiotics while culture and sensitivity results are awaited.

\section{References}

1. Hooton TM, Stamm WE. Diagnosis and treatment of uncomplicated urinary tract infection. Infect Dis Clin North Am 1997;11(3):551-581.

2. Guneysel O, Onur O, Erdede M, Denizbasi A. Trimethoprim/sulfamethoxazole resistance in urinary tract infections. J Emerg Med 2009;36(4):338-341.

3. Ronald A. The etiology of urinary tract infection: traditional and emerging pathogens. Am J Med 2002;8(113, suppl 1A): 14S-19S

4. Gupta K. Addressing antibiotic resistance. Am J Med 2002;8(113, suppl 1A):29S-34S.

5. Miller LG, Tang AW. Treatment of uncomplicated urinary tract infections in an era of increasing antimicrobial resistance. Mayo Clin Proc 2004;79(8):1048-1053.

6. Sanford J P, Gilbert DN, Moellering Jr RC, Eliopoulos GM, Sande MA, Chambers HF. The Sanfor Guide to Antimicrobial Therapy. 38th ed. Sperryville, VA, 2008: 27 (Table 1), 30.

7. Sobel JD, Kaye D. Urinary tract infections. In: Mandell GL, Bennett JE, Dolin R, eds. Principles and practice of Infectious diseases. 6th ed. Philadelphia, Pa.: Elsevier Churchill Livingstone, 2005: 890.

8. Huovinen P. Resistance to TMP-SMX. Clin Infect Dis 2001;32:1608-1614.

9. Kallen AJ, Welch HG, Sirovich BE. Current antibiotic therapy for isolated urinary tract infections in women. Arch Intern Med 2006;166:635-639.

10. Paterson DL. Resistance in gram-negative bacteria: Enterobacteriaceae. Am J Med 2006;119(6):Suppl 1:S20-28, discussion $\mathrm{S} 62-70$.

11. Pitout JD, Laupland KB. Extended-spectrum beta-lactamase-producing Enterobacteriaceae: an emerging public-health concern. Lancet Infect Dis 2008;8(3):159-166.

12. Woodford N, Ward ME, Kaufmann ME, et al. Community and hospital spread of Escherichia coli producing CTX-M extended-spectrum beta-lactamases in the UK. J Antimicrob Chemothe 2004;54(4):735-743

13. Hillier S, Roberts Z, Dunstan F, Butler C, Howard A, Palmer S. Prior antibiotics and risk of antibioticresistant community-acquired urinary tract infection: a case-control study. J Antimicrob Chemothe 2007;60(1):92-99.

14. Entenza JM, Que YA, Vouillamoz J, Glauser MP, Moreillon P. Efficacies of moxifloxacin, ciprofloxacin, and vancomycin against experimental endocarditis due to methicillin-resistant Staphylococcus aureus expressing various degrees of ciprofloxacin resistance. Antimicrob Agents Chemother 2001;45(11):3076-3083

15. Costelloe C, Metcalfe C, Lovering A, Mant D, Hay AD. Effect of antibiotic prescribing in primary care on antimicrobial resistance in individual patients: systematic review and meta-analysis. BMJ 2010; 340:c2096.

16. Alam MF, Cohen D, Butler C, et al. The additional costs of antibiotics and re-consultations for antibioticresistant Escherichia coli urinary tract infections managed in general practice. Int J Antimicrob Agents 2009;33(3):255-257.

17. Little P, Turner S, Rumsby $\mathrm{K}$, et al. Dipsticks and diagnostic algorithms in urinary tract infection: development and validation, randomised trial, economic analysis, observational cohort and qualitative study. Health Technol Assess 2009;13(19):iii-iv, ix-xi, 1-73.

Accepted 11 October 2010. 